\title{
The Dirac Electron in Simple Fields*
}

\author{
By Milton S. Plesset \\ Sloane Physics Laboratory, Yale University
}

(Received June 6, 1932)

\begin{abstract}
The relativity wave equations for the Dirac electron are transformed in a simple manner into a symmetric canonical form. This canonical form makes readily possible the investigation of the characteristics of the solutions of these relativity equations for simple potential fields. If the potential is a polynomial of any degree in $x$, a continuous energy spectrum characterizes the solutions. If the potential is a polynomial of any degree in $1 / x$, the solutions possess a continuous energy spectrum when the energy is numerically greater than the rest-energy of the electron; values of the energy numerically less than the rest-energy are barred. When the potential is a polynomial of any degree in $r$, all values of the energy are allowed. For potentials which are polynomials in $1 / r$ of degree higher than the first, the energy spectrum is again continuous. The quantization arising for the Coulomb potential is an exceptional case.
\end{abstract}

1.

I N HIS treatment of the reflection of the relativity electron at a potential jump Klein ${ }^{1}$ found a paradoxical behavior of the Dirac electron associated with the possibility of the existence of states of negative kinetic energy. He showed by an ingenious treatment that the reflection coefficient for electrons incident upon a discontinuous potential jump of height $P$ varied with $P$ from the value zero for $P=0$ to the value unity for $P=W-m c^{2}$ ( $W$ being the energy of the incident electrons). For this last value of $P$ the momentum $p$ associated with the transmitted beam had the value zero, and as $P$ was increased beyond $W-m c^{2}$ this momentum became imaginary and the reflection coefficient remained unity until $P$ attained the value $W+m c^{2}$. The results thus far are exactly what would be expected. If $P$ is increased further one enters the domain of negative kinetic energy wherein the group velocity and the momentum in the transmitted beam are oppositely directed; also the reflection coefficient falls off from the value unity and approaches the value $(W-c p) /(W+c p)$ as $P$ is indefinitely increased. Thus by a transition to a state of negative kinetic energy the Dirac electron has apparently an appreciable probability of penetrating a barrier of infinite height. Bohr suggested that this peculiar result might be due to a jump in potential of the order of $m c^{2}$ over a region of the order of the Compton wave-length $h / m c$. It is within a region of the order of $h / m c$ that the internal structure of the Dirac electron and the accompanying "trembling" phenomenon ${ }^{2}$ manifests itself. This supposition of Bohr was verified by Sauter ${ }^{3}$ who treated the problem of the

* The results of this paper were presented at the Washington meeting of the American Physical Society (April, 1932).

${ }^{1}$ O. Klein, Zeits. f. Physik 53, 157 (1929).

2 E. Schrödinger, Preuss. Akad. Wiss. Berlin, Ber. 24, 418 (1930).

${ }^{3}$ F. Sauter, Zeits. f. Physik 69, 742 (1931). 
Dirac electron passing through a potential barrier in which the rise of potential was taken to be linear. Sauter then showed that the transmission coefficient was very small for a rise in potential $P>W+m c^{2}$.

Here we shall discuss analytically the nature of the stationary solutions of Dirac's equations for continuous potential functions of a more general type than those heretofore considered. A simple familiar method of treating these equations will be used. The results secured are perhaps unexpected on the basis of the corresponding results for the nonrelativistic treatment.

\section{2.}

The relativistic equation of $\operatorname{Dirac}^{4}$ for an electron of charge $-e$ is

$$
\begin{aligned}
\left\{\frac{W}{c}+\frac{e}{c} A_{0}\right. & +\alpha_{x}\left(p_{x}+\frac{e}{c} A_{x}\right)+\alpha_{y}\left(p_{y}+\frac{e}{c} A_{y}\right) \\
& \left.+\alpha_{z}\left(p_{z}+\frac{e}{c} A_{z}\right)+\rho_{3} m c\right\} \Psi=0 .
\end{aligned}
$$

We shall suppose that $A=0$; then Eq. (1) may be expanded as follows

$$
\begin{aligned}
& (W / c-V / c+m c) \Psi_{1}+\left(p_{x}-i p_{y}\right) \Psi_{4}+p_{z} \Psi_{3}=0 \\
& (W / c-V / c+m c) \Psi_{2}+\left(p_{x}+i p_{y}\right) \Psi_{3}-p_{z} \Psi_{4}=0 \\
& (W / c-V / c-m c) \Psi_{3}+\left(p_{x}-i p_{y}\right) \Psi_{2}+p_{z} \Psi_{1}=0 \\
& (W / c-V / c-m c) \Psi_{4}+\left(p_{x}+i p_{y}\right) \Psi_{1}-p_{z} \Psi_{2}=0
\end{aligned}
$$

where we have put $V$ for the potential energy $-e A_{0}$. The expansion of (1) into (2) corresponds to these choices of the $\alpha$ 's and $\rho_{3}$

$$
\begin{array}{rlrl}
\alpha_{x} & =\left(\begin{array}{rrrr}
0 & 0 & 0 & 1 \\
0 & 0 & 1 & 0 \\
0 & 1 & 0 & 0 \\
1 & 0 & 0 & 0
\end{array}\right), & \alpha_{y}=\left(\begin{array}{rrrrr}
0 & 0 & 0 & -i \\
0 & 0 & i & 0 \\
0 & -i & 0 & 0 \\
i & 0 & 0 & 0
\end{array}\right), \\
\alpha_{z}=\left(\begin{array}{rrrr}
0 & 0 & 1 & 0 \\
0 & 0 & 0 & -1 \\
1 & 0 & 0 & 0 \\
0 & -1 & 0 & 0
\end{array}\right), & \rho_{3}=\left(\begin{array}{rrrr}
1 & 0 & 0 & 0 \\
0 & 1 & 0 & 0 \\
0 & 0 & -1 & 0 \\
0 & 0 & 0 & -1
\end{array}\right) .
\end{array}
$$

We shall consider first the case for which $V$ is a polynomial ${ }^{5}$ of any degree in $x$,

$$
V=\sum_{n=0}^{q} a_{n} x^{n}, \quad(0<q<\infty)
$$

${ }^{4}$ P. A. M. Dirac, The Principles of Quantum Mechanics, p. 243.

5 The case of a uniform field, $V=a x$, has been treated by Sauter, Zeits. f. Physik 69, 742 (1931), and that of the simple harmonic oscillator, $V=a x^{2}$, by K. Nikolsky, Zeits. f. Physik 62, 677 (1930); the methods used in these cases differ from that used here and are perhaps not so readily capable of generalization as the present method. 
and we shall seek solutions of the form

$$
\Psi=\left(\exp 2 \pi i\left(p_{y} y+p_{z} z-W t\right) / h\right) \psi(x)
$$

so that the components of $\psi(x)$ satisfy these differential equations

$$
\begin{aligned}
& \frac{d \psi_{1}}{d x}=-\frac{2 \pi i}{h}\left\{i p_{y} \psi_{1}-p_{z} \psi_{2}+\left(\frac{W}{c}-\frac{V}{c}-m c\right) \psi_{4}\right\} ; \\
& \frac{d \psi_{2}}{d x}=-\frac{2 \pi i}{h}\left\{p_{z} \psi_{1}-i p_{y} \psi_{2}+\left(\frac{W}{c}-\frac{V}{c}-m c\right) \psi_{3}\right\} ; \\
& \frac{d \psi_{3}}{d x}=-\frac{2 \pi i}{h}\left\{\left(\frac{W}{c}-\frac{V}{c}+m c\right) \psi_{2}+i p_{y} \psi_{3}-p_{z} \psi_{4}\right\} ; \\
& \frac{d \psi_{4}}{d x}=-\frac{2 \pi i}{h}\left\{\left(\frac{W}{c}-\frac{V}{c}+m c\right) \psi_{1}+p_{z} \psi_{3}-i p_{y} \psi_{4}\right\} .
\end{aligned}
$$

In view of (3) it may be said that every finite value of $x$ corresponds to an ordinary point of the system of differential equations (5); hence for all finite values of $x$ the solutions of (5) are not only finite but analytic. The points at infinity, $x= \pm \infty$, are, however, irregular singular points of Eqs. (5) of rank $q+1$. The behavior of the solutions of (5) in the neighborhood of these singular points will now be investigated.

We consider the possibility of formally satisfying Eqs. (5) by a set of normal solutions of the type

$$
\psi_{i}=e^{Q(x)} u_{i}(x), \quad(i=1, \cdots 4)
$$

where $u_{i}(x)$ is assumed to be regular in the neighborhood of $|x|=\infty$, and

$$
Q(x)=\alpha x^{q+1} /(q+1)+\beta x^{q} / q+\cdots+\lambda x .
$$

The permissible values of $\alpha$ are determined by the characteristic equation

$$
\left|\begin{array}{rrrr}
-\alpha & 0 & 0 & a \\
0 & -\alpha & a & 0 \\
0 & a & -\alpha & 0 \\
a & 0 & 0 & -\alpha
\end{array}\right|=0 ; \quad a=2 \pi i a_{q} / h c .
$$

The roots of this equation, which are both double, are

$$
\alpha= \pm a= \pm 2 \pi i \cdot a_{q} / h c
$$

the fact that there are only two distinct roots of Eq. (8) indicates that there are only two independent sets of solutions of the system of Eqs. (5). We transform Eqs. (5) into an analytically symmetrical form by the simple transformation

$$
\begin{aligned}
& \psi_{1}{ }^{\prime}=\left(1 / 2^{1 / 2}\right)\left(\psi_{1}+\psi_{4}\right) ; \\
& \psi_{2}{ }^{\prime}=\left(1 / 2^{1 / 2}\right)\left(\psi_{2}+\psi_{3}\right) ; \\
& {\psi_{3}}^{\prime}=\left(1 / 2^{1 / 2}\right)\left(\psi_{2}-\psi_{3}\right) ; \\
& \psi_{4}{ }^{\prime}=\left(1 / 2^{1 / 2}\right)\left(\psi_{1}-\psi_{4}\right) .
\end{aligned}
$$


The matrix of the constants in the linear transformation (10)

$$
C=\left(\begin{array}{cccc}
1 / 2^{1 / 2} & 0 & 0 & 1 / 2^{1 / 2} \\
0 & 1 / 2^{1 / 2} & 1 / 2^{1 / 2} & 0 \\
0 & 1 / 2^{1 / 2} & -1 / 2^{1 / 2} & 0 \\
1 / 2^{1 / 2} & 0 & 0 & -1 / 2^{1 / 2}
\end{array}\right)=C^{-1}
$$

generates a unitary transformation of the matrix of the determinant of the characteristic equation in to the diagonal form

$$
\left(\begin{array}{cccc}
-\alpha+a & 0 & 0 & 0 \\
0 & -\alpha+a & 0 & 0 \\
0 & 0 & -\alpha-a & 0 \\
0 & 0 & 0 & -\alpha-a
\end{array}\right)
$$

The "canonical" form of the system of Eqs. (5) is

$$
\begin{aligned}
& \frac{d \psi_{1}{ }^{\prime}}{d x}=-\frac{2 \pi i}{h}\left\{\left(\frac{W}{c}-\frac{V}{c}\right) \psi_{1}{ }^{\prime}-p_{z} \psi_{3}{ }^{\prime}+\left(m c+i p_{y}\right) \psi_{4}{ }^{\prime}\right\} \\
& \frac{d \psi_{2}^{\prime}}{d x}=-\frac{2 \pi i}{h}\left\{\left(\frac{W}{c}-\frac{V}{c}\right) \psi_{2}{ }^{\prime}+\left(m c-i p_{y}\right) \psi_{3}{ }^{\prime}+p_{z} \psi_{4}^{\prime}\right\} \\
& \frac{d \psi_{3}^{\prime}}{d x}=-\frac{2 \pi i}{h}\left\{p_{z} \psi_{1}^{\prime}-\left(m c+i p_{y}\right) \psi_{2}^{\prime}-\left(\frac{W}{c}-\frac{V}{c}\right) \psi_{3}{ }^{\prime}\right\} \\
& \frac{d \psi_{4}^{\prime}}{d x}=-\frac{2 \pi i}{h}\left\{\left(i p_{y}-m c\right) \psi_{1}^{\prime}-p_{z} \psi_{2}^{\prime}-\left(\frac{W}{c}-\frac{V}{c}\right) \psi_{4}^{\prime}\right\} .
\end{aligned}
$$

We are assuming solutions of this system of Eqs. (13) of the form (an additional subscript $s$ is introduced to distinguish the two sets of solutions)

$$
\begin{array}{lll}
\psi_{1 s}{ }^{\prime}=e^{Q_{s}(x)} x^{\mu_{s}} B_{1 s}(x) ; & \psi_{2 s}{ }^{\prime}=e^{Q_{s}(x)} x^{\mu_{s}} B_{2 s}(x) ; & (s=1,2) \\
\psi_{3 s}{ }^{\prime}=e^{Q_{s}(x)} x^{\mu_{s}} B_{3 s}(x) ; & \psi_{4 s}{ }^{\prime}=e^{Q_{s}(x)} x^{\mu_{s}} B_{4 s}(x) ; &
\end{array}
$$

where

$$
Q_{s}(x)=\alpha_{s} x^{q+1} /(q+1)+\beta_{s} x^{q} / q+\cdots+\lambda_{s} x
$$

and

$$
B_{r s}(x)=B_{r s}{ }^{0}+B_{r s}{ }^{(1)} x^{-1}+B_{r s}^{(2)} x^{-2}+\cdots .
$$

The constants of the solutions (14) are evaluated by direct substitution into Eqs. (13). Of the two sets of formal solutions the first may be written as

$$
\begin{aligned}
& \psi_{11}{ }^{\prime}=e^{Q_{1}(x)}\left\{B_{11}{ }^{0}+B_{11}^{(q-1)} / x^{q-1}+\cdots\right\} \\
& \psi_{21}{ }^{\prime}=e^{Q_{1}(x)}\left\{B_{21^{0}}+B_{21}(q-1) / x^{q-1}+\cdots\right\} \\
& \psi_{31}{ }^{\prime}=e^{Q_{1}(x)}\left\{B_{31}{ }^{(q)} / x^{q}+\cdots\right\} \\
& \psi_{41}{ }^{\prime}=e^{Q_{1}(x)}\left\{B_{41}{ }^{(q)} / x^{q}+\cdots\right\}
\end{aligned}
$$


where

$$
Q_{1}(x)=\frac{2 \pi i}{h c} \sum_{n=0}^{q} \frac{a_{n} x^{n+1}}{n+1}-\frac{2 \pi i W x}{h c} .
$$

It may be shown by examination of the recurrence relations which determine the $B$ 's that $\psi^{\prime}{ }_{11}$ and $\psi_{21}{ }^{\prime}$ are linearly dependent as are also $\psi^{\prime}{ }_{31}$ and $\psi_{41}^{\prime}$. The second set of solutions is

where

$$
\begin{aligned}
& \psi_{12}{ }^{\prime}=e^{Q_{2}(x)}\left\{B_{12}{ }^{(q)} / x^{q}+\cdots\right\} \\
& \psi_{22}{ }^{\prime}=e^{Q_{2}(x)}\left\{B_{22}{ }^{(q)} / x^{q}+\cdots\right\} \\
& \psi_{32}{ }^{\prime}=e^{Q_{2}(x)}\left\{B_{32^{0}}+B_{32}{ }^{(q-1)} / x^{q-1}+\cdots\right\} \\
& {\psi_{42}}^{\prime}=e^{Q_{2}(x)}\left\{B_{42}{ }^{0}+B_{42}{ }^{(q-1)} / x^{q-1}+\cdots\right\}
\end{aligned}
$$

$$
Q_{2}(x)=-\frac{2 \pi i}{h c} \sum_{n=0}^{q} \frac{a_{n} x^{n+1}}{n+1}+\frac{2 \pi i W x}{h c} .
$$

Again $\psi_{12}{ }^{\prime}$ and $\psi_{22}{ }^{\prime}$ are linearly dependent as are also $\psi_{32}{ }^{\prime}$ and $\psi_{42}{ }^{\prime}$. We have further that only the constants $B_{11}{ }^{0}, B_{21}{ }^{0}, B_{32}{ }^{0}, B_{42}{ }^{0}$ are arbitrary.

We wish to use the solutions (17) and (18), which we have found to satisfy the canonical equations (13) formally, to discuss the behavior of the true solutions for values of $x$ numerically large; this use requires a rigorous justification since it follows from the recurrence relations for the $B$ 's that the infinite series $B_{r s}(x)$ do not in general converge for finite values of $x$. This justification might be carried out by eliminating as follows from the canonical Eqs. (13). Put

$$
\begin{array}{ll}
\psi_{1}{ }^{\prime}=e^{Q_{1}(x)} u_{1}(x) ; & \psi_{2}{ }^{\prime}=e^{Q_{1}(x)} u_{2}(x) ; \\
\psi_{3}{ }^{\prime}=e^{Q_{1}(x)} u_{3}(x) ; & \psi_{4}{ }^{\prime}=e^{Q_{1}(x)} u_{4}(x)
\end{array}
$$

where $Q_{1}(x)$ is the polynomial given above in (17a). Then the simultaneous Eqs. (13) give readily

$$
\begin{aligned}
\frac{d^{2} u_{3}}{d x^{2}}+\frac{4 \pi i}{h c}\{V(x)-W\} & \frac{d u_{3}}{d x} \\
& +\frac{4 \pi}{h c}\left\{i \frac{d V}{d x}-\frac{\pi c}{h}\left(p_{y}{ }^{2}+p_{z}{ }^{2}+m^{2} c^{2}\right)\right\} u_{3}=0 ;
\end{aligned}
$$

$u_{4}$ satisfies the same equation as $u_{3}$. We get also

$$
\frac{d^{2} u_{1}}{d x^{2}}+\frac{4 \pi i}{h c}\{V(x)-W\} \frac{d u_{1}}{d x}-\frac{4 \pi^{2}}{h^{2}}\left\{p y^{2}+p_{z}{ }^{2}+m^{2} c^{2}\right\} u_{1}=0 ;
$$

and $u_{2}$ satisfies the same equation as $u_{1}$. The symmetry and simplicity with which elimination can be accomplished to give Eqs. (20) and (21) show the analytical advantage of working with the canonical form of Dirac's equations. If elimination were attempted directly upon Eqs. (5), the result would be an extremely unsymmetrical set of equations. ${ }^{6}$ Eqs. (20) and (21) show that the

${ }^{6}$ This analytical lack of symmetry holds also for Weyl's expansion of Dirac's equation. 
points $x= \pm \infty$ are irregular singular points of the functions $u_{i}(x)$ as well as of the functions $\psi_{i}{ }^{\prime}(x)$. Instead of proceeding further with Eqs. (20) and (21), it is more convenient to treat the canonical Eqs. (13) directly. These equations, now considered in the complex plane, have as solutions the generalized Laplace contour integrals

$$
\begin{gathered}
\psi_{r s}{ }^{\prime}=\left(\exp \beta_{s} z^{q} / q+\cdots+\lambda_{s} z\right) \int_{C_{s}}\left(\exp z^{q+1}\right)\left(\zeta-\frac{\alpha_{s}}{q+1}\right)^{-\left(\mu_{s}+1\right) /(q+1)} \\
\sum_{k=0}^{q} z^{k} \phi_{r k}{ }^{(s)}\left(\zeta-\frac{\alpha_{s}}{q+1}\right) d \zeta
\end{gathered}
$$

The contour $C_{s}$ is a loop circuit about $\zeta=\alpha_{s} /(q+1)$ such that the real part of $\left[z^{q+1}\left\{\zeta-\alpha_{s} /(q+1)\right\}\right]$ is negative along its ray. The functions $\phi_{r k}^{(\mathrm{s})}$ are analytic functions (determined by (13)) in the neighborhood of $\zeta=\alpha_{s} /(q+1)$. These contour integrals (22) are precise solutions of the canonical equations; the asymptotic expansions $\mathbf{s}^{7}$ of these contour integrals, valid in certain sectors of the $z$-plane which may be so taken as to include the real axis, are exactly the formal solutions (17) and (18) written above. ${ }^{8}$ Therefore these formal solutions have significance as the asymptotic solutions of Eqs. (13). Their use in discussing the behavior of the solutions of (13) for numerically large values of $x$ is thus justified. The importance of using the canonical form of Dirac's equations is here apparent; for, if formal solutions of the type (6) were attempted with the original Eqs. (5), a divergent expansion would result which would be meaningless.

We may now say from Eqs. (17), (17a) and (18), (18a) that the components of the Dirac wave function, remain finite as $x$ becomes infinite; further we have

$$
\int_{-a}^{a} \sum_{1}^{4} \psi_{i} \psi_{i}^{*} d x<c(a)
$$

where $c$ is a finite number, since the functions $\psi_{i}$ are finite and analytic for all finite values of $x$. And if the constant $a$ is sufficiently large $(b>a)$,

$$
\int_{a}^{b} \sum_{1}^{4} \psi_{i} \psi_{i} * d x \propto(b-a) ; \quad \int_{-a}^{-b} \sum_{1}^{4} \psi_{i} \psi_{i} * d x \propto-(b-a) ;
$$

that is, the integral square becomes proportional to the domain of integration. The wave functions exhibit, therefore, quite common characteristics of the wave functions associated with a continuous energy spectrum. It is thus clear that the Dirac electron possesses a continuous spectrum with all values of $W$ allowed for a potential field which is a polynomial of any degree in $x$.

\section{3.}

Let us now consider potential fields which are polynomials in $1 / x$,

$$
V(x)=\sum_{n=0}^{q} a_{n} x^{-n} . \quad(0<q<\infty)
$$

${ }^{7}$ Asymptotic expansions in the sense of Poincaré.

8 Cf. E. L. Ince, Ordinary Differential Equations p. 484. 
It is evident from the discussion given above that we may make the simplifying assumption $p_{y}=p_{z}=0$ in Eqs. (5) without affecting the generality of the results secured. Then Eqs. (5) become

$$
\begin{aligned}
& \frac{d \psi_{1}}{d x}=-\frac{2 \pi i}{h c}\left(W-m c^{2}-V\right) \psi_{4} ; \\
& \frac{d \psi_{2}}{d x}=-\frac{2 \pi i}{h c}\left(W-m c^{2}-V\right) \psi_{3} ; \\
& \frac{d \psi_{3}}{d x}=-\frac{2 \pi i}{h c}\left(W+m c^{2}-V\right) \psi_{2} ; \\
& \frac{d \psi_{4}}{d x}=-\frac{2 \pi i}{h c}\left(W+m c^{2}-V\right) \psi_{1} .
\end{aligned}
$$

The complete behavior of this system of equations may be determined by considering only Eqs. (26a) and (26d) since this pair of equations has the same form as the pair of Eqs. (26b) and (26c). For the potential function given in (25) the system of Eqs. (26) has an irregular singularity at $x= \pm \infty$. of rank unity and an irregular singularity at $x=0$ of rank $q-1$.

To investigate the behavior of the solutions in the neighborhood of the origin we proceed by the method given in $\$ 2$ above. The transformation to the canonical form of (26a) and (26d) is given by

$$
\begin{aligned}
& \psi_{1}{ }^{\prime}=\left(1 / 2^{1 / 2}\right)\left(\psi_{1}+\psi_{4}\right), \\
& {\psi_{2}}^{\prime}=\left(1 / 2^{1 / 2}\right)\left(\psi_{1}-\psi_{4}\right),
\end{aligned}
$$

so that Eqs. (26a) and (26d) become

$$
\begin{aligned}
& \frac{d \psi_{1}{ }^{\prime}}{d x}=-\frac{2 \pi i}{h c}\left\{[W-V(x)] \psi_{1}{ }^{\prime}+m c^{2} \psi_{2}{ }^{\prime}\right\} ; \\
& \frac{d \psi_{2}^{\prime}}{d x}=\frac{2 \pi i}{h c}\left\{m c^{2} \psi_{1}{ }^{\prime}+[W-V(x)] \psi_{2}{ }^{\prime}\right\} .
\end{aligned}
$$

To make the analytical procedure of $\$ 2$ completely applicable we need only put $x=1 / y$ in Eqs. (28) to get

$$
\begin{aligned}
& \frac{d \psi_{1}{ }^{\prime}}{d y}=\frac{2 \pi i}{h c} \frac{1}{y^{2}}\left\{\left[W-V\left(\frac{1}{y}\right)\right] \psi_{1}{ }^{\prime}+m c^{2} \psi_{2}{ }^{\prime}\right\} \\
& \frac{d \psi_{2}{ }^{\prime}}{d y}=-\frac{2 \pi i}{h c} \frac{1}{y^{2}}\left\{m c^{2} \psi_{1}{ }^{\prime}+\left[W-V\left(\frac{1}{y}\right)\right] \psi_{2}{ }^{\prime}\right\} .
\end{aligned}
$$

We now consider the behavior of the solutions of Eqs. (29) for values of $y$ numerically large. We assume, as before, formal solutions of the normal type

$$
\psi_{r s}{ }^{\prime}=e^{Q_{s}(y)} y^{\mu_{s}} B_{r s}(y) \quad(r, s=1,2)
$$


where now

$$
Q_{s}(y)=\frac{\alpha_{s} y^{q-1}}{q-1}+\frac{\beta_{s} y^{q-2}}{q-2}+\cdots+\lambda_{s} y
$$

and as before

$$
B_{r s}(y)=B_{r s}{ }^{0}+B_{r s}{ }^{(1)} y^{-1}+\cdots .
$$

By direct substitution it is found that the asymptotic solutions have the form

$\psi_{r s}{ }^{\prime}(y) \cong \delta_{r s} \exp \left\{(-1)^{s \frac{2 \pi i}{h c}}\left[\frac{a_{q}}{q-1} y^{q-1}+\frac{a_{q-2}}{q-2} y^{q-2}+\cdots+a_{1} \log y\right]\right\}$

where $\delta_{r s}$ is the Kronecker delta. From Eq. (31) it is clear that the solutions of Eqs. (29) remain finite as $y$ approaches infinity for all values of $W$; that is, the solutions of Eqs. (28) remain finite in the neighborhood of $x=0$. The origin, $x=0$, does not, therefore, give any limitations on possible allowed solutions of Eqs. (28) or (26).

We must now investigate the behavior of the solutions of Eqs. (26a) and (26d) near $x= \pm \infty$. The characteristic equation for (26a) and (26d) is

$$
\left|\begin{array}{cc}
-\alpha & -\frac{2 \pi i}{h c}\left(W-m c^{2}-a_{0}\right) \\
-\frac{2 \pi i}{h c}\left(W+m c^{2}-a_{0}\right) & -\alpha
\end{array}\right|=0 ;
$$

so that

$$
\alpha= \pm(2 \pi / h c)\left\{m^{2} c^{4}-\left(W-a_{0}\right)^{2}\right\}^{1 / 2} .
$$

We may secure a canonical form of Eqs. (26a) and (26d) by putting

where

$$
\begin{aligned}
& \psi_{1}=a_{1}{ }^{1 / 2}\left(\psi_{1}{ }^{\prime}-\psi_{2}{ }^{\prime}\right) \\
& \psi_{4}=a_{2}{ }^{1 / 2}\left(\psi_{1}{ }^{\prime}+\psi_{2}{ }^{\prime}\right)
\end{aligned}
$$

$$
\frac{1}{a_{1}}=\left(W+m c^{2}-a_{0}\right) ; \quad \frac{1}{a_{2}}=\left(W-m c^{2}-a_{0}\right) .
$$

Eqs. (26a) and (26d) then become

$$
\begin{aligned}
\frac{d \psi_{1}{ }^{\prime}}{d x}= & -\frac{2 \pi}{h c}\left\{\left[m^{2} c^{4}-\left(W-a_{0}\right)^{2}\right]^{1 / 2}+\frac{\left(W-a_{0}\right)}{\left[m^{2} c^{4}-\left(W-a_{0}\right)^{2}\right]^{1 / 2}}\left(V-a_{0}\right)\right\} \psi_{1}{ }^{\prime} \\
& -\frac{2 \pi}{h c} \frac{m c^{2}}{\left[m^{2} c^{4}-\left(W-a_{0}\right)^{2}\right]^{1 / 2}}\left(V-a_{0}\right) \psi_{2}{ }^{\prime} ; \\
\frac{d \psi_{2}^{\prime}}{d x}= & \frac{2 \pi}{h c} \frac{m c^{2}}{\left[m^{2} c^{4}-\left(W-a_{0}\right)^{2}\right]^{1 / 2}}\left(V-a_{0}\right) \psi_{1}{ }^{\prime} \\
+ & \frac{2 \pi}{h c}\left\{\left[m^{2} c^{4}-\left(W-a_{0}\right)^{2}\right]^{1 / 2}+\frac{\left(W-a_{0}\right)}{\left[m^{2} c^{4}-\left(W-a_{0}\right)^{2}\right]^{1 / 2}}\left(V-a_{0}\right)\right\} \psi_{2}{ }^{\prime} .
\end{aligned}
$$


The formal substitution into Eqs. (35) of solutions of the normal type gives the asymptotic solutions of these equations. The asymptotic solutions are readily found to be

$$
\psi_{r s}{ }^{\prime} \cong e^{\alpha_{s} x} x^{\mu s} \delta_{r s} \quad(r, s=1,2)
$$

where

$$
\alpha_{1}=-\frac{2 \pi}{h c}\left[m^{2} c^{4}-\left(W-a_{0}\right)^{2}\right]^{1 / 2}=-\alpha_{2},
$$

and

$$
\mu_{1}=-\frac{2 \pi}{h c} \frac{\left(W-a_{0}\right) a_{1}}{\left[m^{2} c^{4}-\left(W-a_{0}\right)^{2}\right]^{1 / 2}}=-\mu_{2} .
$$

If $\left(W-a_{0}\right)^{2}>m^{2} c^{4}$, it is evident from Eq. (36) that the solutions of Eqs. (35) are all finite at infinity; all such values of $W$ are allowed so that a continuous spectrum arises. If, however, $\left(W-a_{0}\right)^{2}<m^{2} c^{4}$, Eq. (36) shows that it is not possible to find solutions of (35) which remain finite both at $x=+\infty$ and at $x=-\infty$. Hence there are no proper solutions in this band; these energy values are barred.

\section{4.}

We now turn to the case in which the potential function depends on $r$ alone. To examine the characteristics of the Dirac equations for this case we may begin with the radial equations in the form in which they are given by Dirac $^{9}$

$$
\begin{aligned}
& \frac{d R_{a}}{d r}=\frac{j}{r} R_{a}+\left(b_{2}+\frac{2 \pi}{h c} V(r)\right) R_{b} \\
& \frac{d R_{b}}{d r}=-\frac{j}{r} R_{b}+\left(b_{1}-\frac{2 \pi}{h c} V(r)\right) R_{a}
\end{aligned}(j= \pm 1, \pm 2, \cdots)
$$

where

$$
b_{1}=2 \pi\left(m c^{2}+W\right) / h c ; b_{2}=2 \pi\left(m c^{2}-W\right) / h c .
$$

The type of potential to be considered first is that for which $V$ is a polynomial of any degree in $r$

$$
V=\sum_{n=0}^{q} a_{n} r^{n} . \quad(0<q<\infty)
$$

With $V(r)$ given by (40) Eqs. (39) for $R_{a}$ and $R_{b}$ have an irregular singularity at $r=\infty$ of rank $q+1$; the origin, $r=0$, is a regular singular point; and all other points are ordinary points. We consider first the nature of the solutions in the neighborhood of $r=\infty$. The characteristic equation is

9 Dirac, The Principles of Quantum Mechanics, p. 252. 


$$
\left|\begin{array}{cc}
-\alpha & 2 \pi a_{q} / h c \\
-2 \pi a_{q} / h c & -\alpha
\end{array}\right|=0 ; \alpha= \pm \frac{2 \pi i}{h c} a_{q} .
$$

The transformation to a canonical form is given by

$$
\begin{aligned}
& R_{a}=\left(1 / 2^{1 / 2}\right)\left(\psi_{1}-i \psi_{2}\right) \\
& R_{b}=\left(1 / 2^{1 / 2}\right)\left(-i \psi_{1}+\psi_{2}\right)
\end{aligned}
$$

and the canonical form of Eqs. (39) is

$$
\begin{aligned}
& \frac{d \psi_{1}}{d r}=\frac{2 \pi i}{h c}\{W-V\} \psi_{1}+\left\{\frac{2 \pi m c}{h}-i \frac{j}{r}\right\} \psi_{2} ; \\
& \frac{d \psi_{2}}{d r}=\left\{\frac{2 \pi m c}{h}+i \frac{j}{r}\right\} \psi_{1}-\frac{2 \pi i}{h c}\{W-V\} \psi_{2} .
\end{aligned}
$$

The asymptotic solutions of Eqs. (43) may be readily found to be

$$
\psi_{m s} \cong \delta_{m s} \exp \left\{(-1)^{s} \frac{2 \pi i}{h c}\left[\sum_{n=0}^{q} \frac{a_{n}}{n+1} r^{n+1}-W r\right]\right\} . \quad(m, s=1,2) .
$$

From (44) it is seen that the solutions of Eqs. (43) remain finite as $r$ approaches infinity and exhibit an integral square behavior like that discussed above. The irregular singular point at infinity, therefore, does not contribute any limitation on permitted energy values.

The singularity at the origin is regular so that in the neighborhood of the origin solutions of the form

$$
\begin{aligned}
& R_{a s}=r^{\alpha_{s}}\left(B_{a s}{ }^{0}+B_{a s}{ }^{(1)} r+B_{a s}{ }^{(2)} r^{2}+\cdots\right) \\
& R_{b s}=r^{\alpha_{s}}\left(B_{b s}{ }^{0}+B_{b s}{ }^{(1)} r+B_{b s}{ }^{(2)} r^{2}+\cdots\right)
\end{aligned}
$$

satisfy the radial Eqs. (39). From the permissible values of $\alpha_{s}$ as determined by the characteristic equation for this case, $\alpha= \pm j$, it is evident that there is always one solution finite at the origin. Combining this result with that given in (44) we may say that there is always a solution of the radial equations which for all values of $W$ is finite everywhere and which possesses in addition a well behaved integral square for large values of $r$. Hence for potential functions which are polynomials of any degree in $r$ the equations of Dirac give a continuous spectrum with all values of $W$ allowed.

\section{5.}

Let us consider finally the case for which the potential function $V(r)$ is a polynomial in $1 / r$

$$
V(r)=\sum_{n=0}^{q} a_{n} r^{-n} . \quad(0<q<\infty)
$$

With this potential function the radial Eqs. (39) have an irregular singularity at $r=\infty$ of rank unity; the roots of the characteristic equation are

$$
\alpha_{1}=(2 \pi / h c)\left[m^{2} c^{4}-\left(W-a_{0}\right)^{2}\right]^{1 / 2}=-\alpha_{2} .
$$


The transformation

$$
\begin{aligned}
& R_{a}=\left(b_{2}+2 \pi a_{0} / h c\right)^{1 / 2}\left(\psi_{1}-\psi_{2}\right) \\
& R_{b}=\left(b_{1}-2 \pi a_{0} / h c\right)^{1 / 2}\left(\psi_{1}+\psi_{2}\right)
\end{aligned}
$$

gives the canonical form

$$
\begin{aligned}
\frac{d \psi_{1}}{d r}= & \frac{2 \pi}{h c}\left\{\left[m^{2} c^{4}-\left(W-a_{0}\right)^{2}\right]^{1 / 2}+\frac{\left(W-a_{0}\right)}{\left[m^{2} c^{4}-\left(W-a_{0}\right)^{2}\right]^{1 / 2}}\left(V-a_{0}\right)\right\} \psi_{1} \\
& +\left\{-\frac{j}{r}+\frac{2 \pi}{h} \frac{m c}{\left[m^{2} c^{4}-\left(W-a_{0}\right)^{2}\right]^{1 / 2}}\left(V-a_{0}\right)\right\} \psi_{2} ; \\
\frac{d \psi_{2}}{d r}= & -\left\{\frac{j}{r}+\frac{2 \pi}{h} \frac{m c}{\left[m^{2} c^{4}-\left(W-a_{0}\right)^{2}\right]^{1 / 2}}\left(V-a_{0}\right)\right\} \psi_{1} \\
- & \frac{2 \pi}{h c}\left\{\left[m^{2} c^{4}-\left(W-a_{0}\right)^{2}\right]^{1 / 2}+\frac{\left(W-a_{0}\right)}{\left[m^{2} c^{4}-\left(W-a_{0}\right)^{2}\right]^{1 / 2}}\left(V-a_{0}\right)\right\} \psi_{2} ;
\end{aligned}
$$

and the asymptotic behavior of the solutions of Eqs. (49) is given by

$$
\psi_{r s} \cong e^{\alpha_{s} r} \mu^{\mu} \delta_{\gamma_{r s}} \quad(r, s=1,2)
$$

where

$$
\mu_{1}=\frac{2 \pi}{h c} \frac{\left(W-a_{0}\right)}{\left[m^{2} c^{4}-\left(W-a_{0}\right)^{2}\right]^{1 / 2}} a_{1}=-\mu_{2} .
$$

It is evident from (47) and (51) that for $\left(W-a_{0}\right)^{2}>m^{2} c^{4}$ all the solutions remain finite as $r$ approaches infinity; and for $\left(W-a_{0}\right)^{2}<m^{2} c^{4}$ there is one finite and one infinite solution at $r=\infty$.

To investigate the singularity at the origin, $r=0$, it is only necessary to make the transformation $r=1 / y$ in the canonical Eqs. (43). If $q=1$, that is to say, if the potential is a Coulomb potential, $y=\infty$ is a regular singular point and regular solutions may be found as follows

$$
\psi_{r s}=y^{\alpha_{s}}\left(B_{r s}{ }^{0}+B_{r s}{ }^{(1)} y^{-1}+B_{r s}^{(2)} y^{-2}+\cdots\right)
$$

where the exponent $\alpha_{s}$ is fixed by the characteristic equation

$$
\left|\begin{array}{cc}
-\alpha-2 \pi i a_{1} / h c & -i j \\
i j & -\alpha+2 \pi i a_{1} / h c
\end{array}\right|=0 ; \alpha= \pm\left(j^{2}-4 \pi^{2} a_{1}^{2} / h^{2} c^{2}\right)^{1 / 2} .
$$

These values of the exponent $\alpha$ are, of course, precisely those found in the treatment of the hydrogen atom. ${ }^{10}$ From (53) it is evident that there is one finite and one infinite solution at $r=0$; also for $r=\infty$ there is one finite and one infinite solution for $\left(W-a_{0}\right)^{2}<m^{2} c^{4}$ (by Eq. (50)). These are exactly the conditions which lead to quantization.

${ }^{10}$ Dirac, p. 253. 
Such is not the case, however, when $q>1$. For these potential functions the origin, $r=0$, is an irregular singularity. The behavior of the solutions may be investigated by means of Eqs. (43) by the same methods as those used previously. The asymptotic solutions are readily found to be

$$
\begin{aligned}
\psi_{r s}(y) \cong \delta_{r s} \exp \left\{(-1)^{s+1}\right. & \frac{2 \pi i}{h c}\left[\frac{a_{q}}{q-1} y^{q-1}\right. \\
& \left.\left.+\frac{a_{q-1}}{q-2} y^{q-2}+\cdots+a_{1} \log y\right]\right\} . \quad(y=1 / r)
\end{aligned}
$$

From (54) it is clear that when $q>1$ all the solutions of (43) remain finite in the neighborhood of $r=0$ for all values of $W$. From Eq. (50) it was seen that there is always one solution finite at infinity for all values of $W$. Combining (50) and (54), we see that a continuous energy spectrum always arises when the potential function is of the form

$$
V(r)=a_{0}+a_{1} / r+a_{2} / r^{2}+\cdots+a_{q} / r^{q} . \quad(q>1)
$$

We may discuss briefly the analytical source of the difference in the results found for the potentials $V=1 / x$ and $V=1 / r$. We note that for the potential $1 / x$ Eqs. (29) retain their canonical form; that is, the highest power of $y$ in the expression for $d \psi_{i}{ }^{\prime} / d y$ occurs in the coefficient of $\psi_{i}{ }^{\prime}$. The general result (31), therefore, remains applicable to this case also. For the potential $V=1 / r$, however, Eqs. (43) (transformed by $r=1 / y$ and considered for $y \sim \infty$ ) do not have the canonical form because of the presence of the terms involving $j$; thus, this potential must be considered apart from those which contain powers of $1 / r$ beyond the first. The general result (54) is not at all applicable to the Coulomb potential.

We may note further here that, if the potential function $V(x)$ is of the form

$$
V(x)=\sum_{n=-p}^{q} a_{n} x^{n}, \quad(0<p, q<\infty)
$$

a continuous spectrum arises with no values of $W$ barred. Similarly, if the potential $V(r)$ contains direct as well as inverse powers of $r$, all values of $W$ are allowed.

6.

By writing the relativity equation of Dirac in a symmetrical canonical form it has been possible to make deductions of a quite general nature regarding the characteristics of the stationary solutions for simple potential fields. The results here presented for the potential fields which are polynomials in $x$ or $r$ give an analytic generalization of the Klein paradox. We may note also that these potential functions will rise by more than $m c^{2}$ over a region of the order of $h / m c$ if sufficiently large values of $x$ or $r$ are taken. ${ }^{11}$ Hence the hypothesis of Bohr advanced to explain the results of Klein is analytically verified for these general cases also.

${ }^{11}$ We are referring now to polynomial potentials of degree higher than the first. 
Such an argument cannot perhaps be so clearly applied to explain the results here presented for potential fields which are polynomials in $1 / x$ or $1 / r$ since with these potentials the steep rises occur toward the origin. For the Coulomb potential by taking sufficiently small values of $r$ a rise in potential of $m c^{2}$ in a region of the order of a Compton wave-length may be secured, and yet this potential gives eminently satisfactory agreement with experiment. If the potential contains higher powers of $1 / r$ as well as $1 / r$, then, as was stated above, continuous spectra result. Here there is striking disagreement with the results of the nonrelativistic Schrödinger theory; for example potentials of the type $a / r-b / r^{2}$ applied to oscillating rotator models have been shown ${ }^{12}$ to give a discrete spectrum in satisfactory agreement with observed spectra.

We may observe, however, from a purely classical point of view that none of the potentials here discussed (except the Coulomb potential and the linear potential $a x$ ) satisfy Laplace's equation. Classically, then, these potentials correspond to a continuous distribution of charge. The relativity theory of the electron is not sufficiently advanced, possibly, to deal with such a complicated physical situation. It should perhaps not be held at fault for giving peculiar results for potential functions which are over-simplified and in that over-simplification correspond to an over-complicated electromagnetic mechanism.

In conclusion the writer takes pleasure in expressing his appreciation to Professor Page for his kind interest in this work. 